\title{
Intelligent Retrieval of AXISYMMETRIC Solid Parts in Machining Process Planning by Case Based Reasoning
}

\author{
Ricardo Sors Lancho \\ Departamento de Manufactura, Fábrica de Cosechadoras de \\ Caña KTP, Holguín, Cuba \\ e-mail: ricardo.sors@ktp.co.cu
}

\begin{abstract}
Alexis Cordovés García
Facultad de Ciencias de la Ingeniería, Universidad

Tecnológica Equinoccial, Santo Domingo, Ecuador e-mail: alexiscordoves60@gmail.com
\end{abstract}

Ricardo Lorenzo Avila Rondon

Escuela de Sistemas de Torreón, Universidad Autónoma de Coahuila, Torreón, México

e-mail: rlar001@yahoo.com

\author{
Rogelio García Muñoz \\ Escuela de Sistemas de Torreón, Universidad Autónoma de \\ Coahuila, Torreón, México \\ e-mail: ragamz@yahoo.com
}

José Antonio Yarza Acuña

Escuela de Sistemas de Torreón, Universidad Autónoma de Coahuila, Torreón, México

e-mail: joseayarza71@hotmail.com

\section{Edwin Esteves Parra}

CE CAD/CAM, Universidad de Holguín, Holguín, Cuba e-mail: edwin@cadcam.uho.edu.cu

\begin{abstract}
Arlys Michel Lastres Aleaga
Facultad de Ciencias de la Ingeniería, Universidad Tecnológica Equinoccial, Santo Domingo, Ecuador e-mail: arlysmichel@gmail.com
\end{abstract}

\begin{abstract}
This research is intended to address the cases retrieval phase related to a CAPP system for axisymmetric solid parts supported by the Case Based Reasoning (CBR). The traits of similarity between two parts were identified from the characteristics of the stock and the finished part. As a result of this work, a function of global similarity metric was obtained, and it incorporates in addition to the geometry of the part and the stock attributes: the type of material, the type of stock and the relationship of the diameter-length dimensions of the part. The results constitute an improvement to the retrieval phase of cases with regard to the retrieval of the most similar case in the repository for the execution of new technical task.
\end{abstract}

\section{Keywords-CAPP System, CBR, Machining Technology}

\section{INTRODUCTION}

Different types of knowledge lead to different types of knowledge-based systems, including: rule-based systems [1], probability-based systems [2], expert systems [3],[4],[5] and case-based systems (CBS) [6], [7], [8], [9].

Case Based Reasoning is based on the process of human reasoning from specific experiences lived before. It is the process of solving new problems based on solutions given to previous problems [10].

In engineering, specifically in the CAD/CAPP/CAM systems, CBR has been successfully used and often related to other techniques of Artificial Intelligence (AI) like Artificial Neural Networks (ANN). All CBR systems comprise the phases of retrieval, reuse (adaptation), review and retention of the new solution [11].
The objective of this work is to address the cases retrieval phase related to a CAPP system for axisymmetric solid parts supported by Case Based Reasoning (CBR). Each case is formed by the part description and its manufacturing technology by machining.

\section{LITERATURE REVIEW}

Hao Yang, Wen F. Lu and Alan C. Lin, [12] developed a CAPP system for generating cut paths by machining of axisymmetric parts called PROCASE. This system has the limitation that when comparing two form features it only checks if they match or not geometrically, so it does not take into account the cutting process required to manufacture the parts. In this research field, the CAPlan/CbC tool can also be mentioned. In this tool, the retrieval of the most similar case is mainly based on the dimensions of the parts. The precision and type of material are not considered; these properties are important for the elaboration of machining process [13].

Moreover, Zuntong Wang, Zhanqiang Liu, Xing Ai [14] proposed a model for the representation and determination of the similarity between parts in high-speed machining. Hsin-chi Chang, Wen F. Lu and Xiaoqing Frank Liu, [15] worked the intelligent retrieval and modification of parts by applying the CBR in the machining process planning of axisymmetric parts. They have introduced significant improvements in comparison to previous systems by considering, besides the geometric shape in the similarity function, the precision, the material and also the history of the cutting process. However, when determining the global 
similarity between two cases, they stated a mathematical expression of similarity which only considers the type of heat treatment and the reached hardness value of the part material.

\section{A. Retrieval phase in the machining process planning by Case-Based Reasoning (CBR)}

CBR systems retrieve a similar case according to the attributes of the case description or traits of similarity. The quality of the approximation improves with the incorporation of knowledge in the specific field of application. To achieve this, the global similarity metric is established as a function that relates the different attributes that describe the case and it is decomposed into local similarity metrics for describing each attribute depending on the nature of its data.

\section{B. Similarity of local metric}

The attributes of the processes are grouped according to the nature of the data: quantitative or numeric and qualitative or non-numeric. Zuntong W. [16] classified the local similarities into four classes: (numeric, independent, value range and dependent).

a) Numerical type

$$
\operatorname{Sim}(x, y)=\frac{1}{1+|x-y|}
$$

In this class, the material hardness (HB) and the surface roughness of each step in the part are taken into account, because these have a numerical value which is known before the machining process.

$$
\begin{aligned}
& \text { b) Independent type. } \\
& 1 \text { si } x=y \\
& \operatorname{Sim}(x, y)=\begin{array}{c}
\text { si } x \neq y
\end{array}
\end{aligned}
$$

These attributes have values that do not interact among themselves and they are qualitative of boolean type. The type of material, the stock, the relationship diameter-length of the part, and the heat treatment have this type of local similarity. For their processing, each of these attributes will be subdivided into the corresponding classes.

c) Value range type: The value range attributes are those in which two different values have the same local similarity and it is defined as:

$$
\operatorname{Sim}(x, y)=f(x, y)
$$

Where $f(x, y)$ is a specific function according to the characteristics of the attribute.

$$
\operatorname{Sim}(x, y)=1-\frac{|x-y|}{M}
$$

In this case, the Calculated Tolerance (upper deviation (D s) - lower deviation (D i)) was grouped into ranges of values. Where $M$ is the maximum value assigned to the attribute, which in this case is 11 .

d) Dependent type: These are attributes that have significant local similarities not only to their own values, but also to the values of other attributes. This type of local similarity is defined as:

$$
\operatorname{Sim}(x, y)=f\left(x, y, x_{1}, y_{1}, x_{2}, y_{2}, \ldots x_{n}, y_{n}\right)
$$

Where: $f\left(x, y, x_{1}, y_{1}, x_{2}, y_{2}, \ldots x_{n}, y_{n}\right)$ is an enumerated function of an attribute and its dependent attributes. The geometric similarity is in this type of local similarity.

In the determination of the geometric similarity, the Peripheral Cutting Index (PCI-2D) is used and it is defined from the peripheral machining of the part [13],[15]. Although various turning schemes can be used, generally the steps are trimmed from the larger diameter to the smaller one and the finished turning is performed at the end of the process. The formation of the Peripheral Cutting Index (PCI2D) is described in the next section.

\section{Peripheral Cutting Index (PCI-2D)}

The arrangement of each step with respect to the larger diameter step will influence the order in which the technological steps are carried out. Based on this observation, the (PCI-2D) is developed considering the diameters and their relative arrangement.

Thus the PCI-2D index: 3-2-1-3-2-4-3 corresponds to the part of figure 1 and indicates that the larger diameter with value (1) has an intermediate position. To each form feature corresponds a digit, the first value (3), followed by (2), which indicates that the step (b) will be elaborated earlier than (a) from the larger diameter step (c) with digit equals to 1. In the steps located to the right of the largest step (c) it was preceded in the same way, which shows that the noncontiguous steps (d) and (g) have the same digit (3), that is, they may have the same priority when they are machined if two variants were used for this case (c-e-g-d-f) or (c-e-d-g-f).

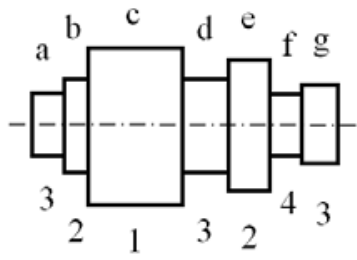

PCI-2D: $3-2-1-3-2-4-3$

Figure 1. Value of Peripheral Cutting Index

The PCI-2D index contains important information for determining the geometric similarity between two parts and it has a generic nature, especially because the new part has not yet been processed technologically. However, after obtaining the relative arrangement among the diameters of the new part, it is necessary to determine the similarity of the geometric configuration of each corresponding step of the parts which have been compared. This similarity is determined by the possible location in the step of key seat, grooves, holes, among other elements (see Figure 5). In this way, the local geometric similarity between the prototype part and the new part will be determined as the sum of the similarities between steps whose values of PCI-2D match.

\section{Global similarity}

The global similarity function in a CBR system integrates the traits of local similarity that describe the specific task for which the system was created. In the case of the system for Process Planning (CAPP) which is presented, the traits of similarity were arranged into two groups. The 
first group characterizes the local geometric similarity of the primitive form features (PFF) of the part and its precision, and the second includes the factors that characterize the material used, its heat treatment and the stock from which the finished part is obtained.

Hsin-chi Chang and Wen F. Lu [15], stated a function of local geometric similarity between two primitive form features, which relates the surface roughness, the type of accuracy and the geometric shape:

$$
\operatorname{Simg}\left(E F_{\mathrm{pi}}, E F_{\mathrm{ni}}\right)=\frac{\operatorname{Sim}_{R z}+\operatorname{Sim}_{I T}}{2} * \operatorname{Sim}_{\mathrm{fg}}
$$

Where: $\operatorname{Sim}_{R z}$ : Local similarity of the superficial roughness between two PFF. Sim IT $_{I T}$ : Local similarity of the tolerance between two PFF. Sim $f g$ : Local similarity of the geometric shape between two PFF.

Local similarity of the geometric shape fg between two PFF, will consider the machining operations to be performed, and how the machining operations are added or eliminated to convert a prototype part in the new part.

Thus:

$$
\operatorname{Sim}_{f g}=W_{p} \frac{\left(T_{p}-L_{p}\right)}{T_{p}}+W_{n} \frac{\left(T_{n}-L_{n}\right)}{T_{n}}
$$

Where: The first term $\boldsymbol{W}_{\boldsymbol{p}} \frac{\left(\boldsymbol{T}_{\boldsymbol{p}}-\boldsymbol{L}_{\boldsymbol{p}}\right)}{\boldsymbol{T} \boldsymbol{p}}$ describes the eliminating operations. The second term $\boldsymbol{W}_{n} \frac{\left(\boldsymbol{T}_{n}-\boldsymbol{L}_{n}\right)}{\boldsymbol{T}_{n}}$ describes the adding operations.

$\boldsymbol{W}_{\boldsymbol{p}} \boldsymbol{y} \boldsymbol{W}_{\boldsymbol{n}}$ : "weight" factors corresponding to the adding and eliminating operations in the prototype part and in the new part, it is assumed here that $\mathrm{W} \_\mathrm{n}<\mathrm{W} \_\mathrm{p}$ because by eliminating cutting operations, of a retrieved case as the most similar, is much easier than adding one from another case which is in the library of cases.

The similarity between two parts, which is determined by the traits of similarity of the second group corresponding to the material, will depend on the following factors or attributes:

$$
\begin{gathered}
\text { Simm (Prototype, NewPart) }= \\
f(\text { Mtt }, \mathrm{HB}, \mathrm{Tm}, \mathrm{Fm}, \mathrm{Dm})
\end{gathered}
$$

Where: (Mtt): Type of heat treatment.(HB): Material hardness. (Tm): Type of Material. (Fm): Form features of the stock. (Dm): Size of the stock, expressed as the relationship between its length and diameter, L/D.

As seen in equation 8, in addition to the factors considered in previous researches, the inclusion of the type of part material, the type of stock and its size, is a contribution of this research in this field.

The factors (Mtt, HB, Tm, Fm, Dm) are qualitative of Boolean type. For example, when comparing two parts that have equal material type, the factor Tm will have a value of 1 , while otherwise it will assume 0 . In the same way, the values will be assigned to each of these factors by the classification established for each factor according to the corresponding standards.

Finally, the global similarity (S) will be determined as follows:

$$
\begin{array}{r}
S=W f \frac{\sum_{i=1}^{k} \operatorname{Simg}(E F p i, E F n i)}{\frac{E F p+E F n}{2}}+ \\
W m \frac{\sum \operatorname{Simm}(M t t, H B, T m, F m, D m)}{\sum M m}
\end{array}
$$

Where: Wf and Wm: Weight factors of the geometric shape and the material, their sum must be equal to 1 .

$\sum_{i=1}^{k} \operatorname{Sim}_{g}(E F p i, E F n i)$ : Is the local geometric similarity, $\mathrm{k}$ is the number of common digits. EFpi is the form feature $\mathbf{i}$ of the prototype part. EFni: Is the form feature $\mathbf{i}$ of the new part. $(E F p+E F n) / 2$ : Are the elements number of the PCI$2 \mathrm{D}$ indexes of the compared parts. $\sum \operatorname{Sim}_{m}\left(M t t, H B, T_{m}, F_{m}, D_{m}\right)$ : Is the similarity of the material attributes of the finished part and the stock. $\sum M m$ : Is the number of the material properties being analyzed. For this case it is equal to 5 .

\section{MATERIALS AND METHODS}

For the development of this work, the information of axisymmetric solid parts made in the machine workshop of the "Combinadas Cañeras" company (KTP) was used. This information has been previously stored in a base of cases with table format of a relational database management system by using the Microsoft Access Database Management System (DBMS). The base of cases is synchronized with the parameters that describe the parts when they were treated by the CAD system, which in this case was AutoCAD 2015. There is also a set of plaintext files in DXF format that are external to the DBMS. This format is already common in most existing commercial CAD systems today, for this reason, this format was used in this work.

The above information was processed on a Pentium IV computer with the following characteristics: dual core processor with $2.0 \mathrm{GHz}$ speed, 2GB RAM, the hard drive space necessary for its execution was $500 \mathrm{MB}$. The operating system Windows XP 32 bits were used to run the developed computing tool. The developed module was programmed in $\mathrm{C}++$ language.

The method used to retrieve the most similar case contained in a database of axisymmetric solid parts is as follows:

1) Define the traits of similarity that best characterize the parts contained in the base of cases from the point of view of its geometry, characteristics of the material and the stock used.

2) Design the database to store the geometric and technological information of the parts made in the conditions of the mentioned machine workshop. This database constitutes the cases (repository) of the CBR system.

3) Conceive the selection algorithm, in the cases repository, of the prototype part that is the most similar one to a new case. The traits of similarity that were defined previously in 1 will be considered in this algorithm.

\section{A. Selection of the traits of similarity}

When conceiving a CBR system, one of the tasks to be solved is to identify the traits of similarity among cases. These traits are also called relevant attributes. In this 
research the traits of similarity of the parts were defined as follows:

a) Geometric similarity of the form features which comprise the model part. The similarity of two PFF, belonging to two different parts, will depend on its location in the corresponding parts, and the difficulty degree of transforming a shape, into the corresponding shape of the other part.

b) Dimensions and shape of stock: The same technological route is not obtained if the part is made from a rolled bar or a bar section, or from a forged or a melt stock. Heat treatment influences the order of the machining process. It is conveniently located in the process prior to finishing operations.

c) Dimensional description: It covers the definition of each PFF dimensions with the linear, diametral, form and position tolerances, and the value of the surface roughness.

d) Material: The type and hardness (HB) of the material influences in the selection of the tools and in the cutting conditions.

e) Heat treatment: It influences in the order of machining process, and it is conveniently located in the process prior to finishing operations.

\section{B. Applying the retrieval phase to a case study}

Here the case study is a subset of two parts. This subset was selected from the cases repository. Both parts are illustrated in the Figure 2 and Figure 3 respectively and these are possible prototypes depending of some conditions. After selection, the next task is to determine which part, of the two parts, is the most similar to the new one (retrieval phase). The resulting part then is considered a prototype for the new one. After that, the IFG is defined from the succession of PFFs contained in the part, and all are illustrated in Figure 5.

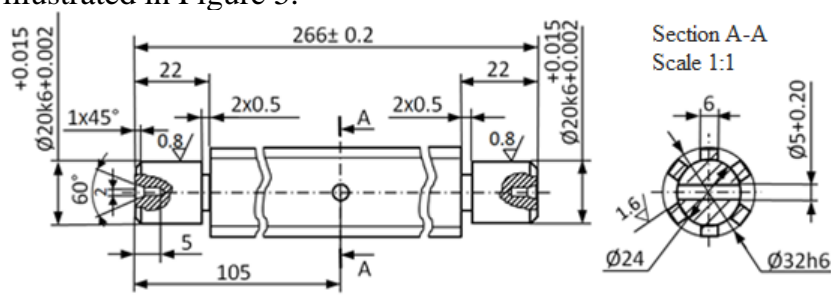

Figure 2. Representation of the case CASE01

IC: CASE01

PCI-2D: 2_1 2 .

IFG: EC25_EE11_EC05

Material: Steel 45

Stock: (Fpb) round-rolled bar (Ø36 X 270 mm), (HB) 197, (TT)-Standardized

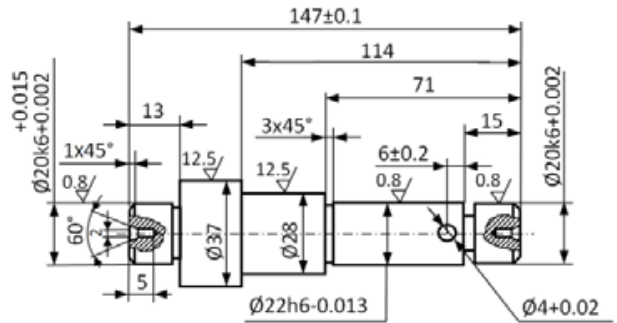

Figure 3. Representation of the case CASE02

IC: CASE02

PCI-2D: 2_1_2_3 4

IFG: EC25_EC45_EC44_EC48_EC05

Material: Steel 45

Stock: (Fpb) round-rolled bar (Ø40 X 152mm), (HB) 197, (TT)-Standardized
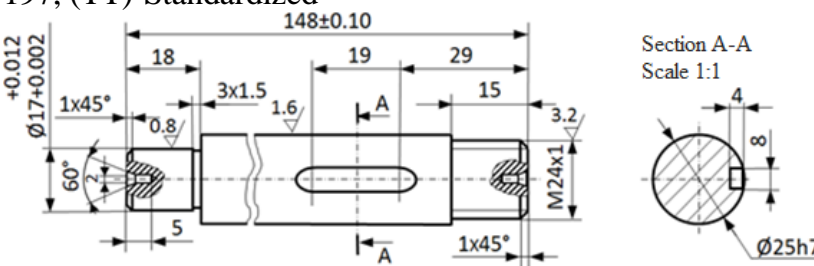

Figure 4. Representation of the new part

PCI-2D for new part: $2 \_1 \_$

IFG: EC25_ECH12_ER11

Material: Steel 45

Stock: (Fpb) round-rolled bar (Ø28 X 203 mm), (HB) 197, (TT)-Standardized

According to equation (9), the calculation of the global similarity between the new part and the two cases, shows that CASE01 is the most similar part to the new part with a similarity value of 0,92 . In Table 1 the new part global similarity values comparison with parts repository is showed.

TABLE 1. NEW PART GLOBAL SIMILARITY VALUES COMPARISON WITH TWO PARTS REPOSITORY

\begin{tabular}{|c|c|}
\hline Comparison & Global similarity \\
\hline New part and CASE01 & 0.92 \\
\hline New part and CASE02 & 0.50 \\
\hline
\end{tabular}

The primitive form features that were used in the parts of the case study are shown in Figure 5 as follows.

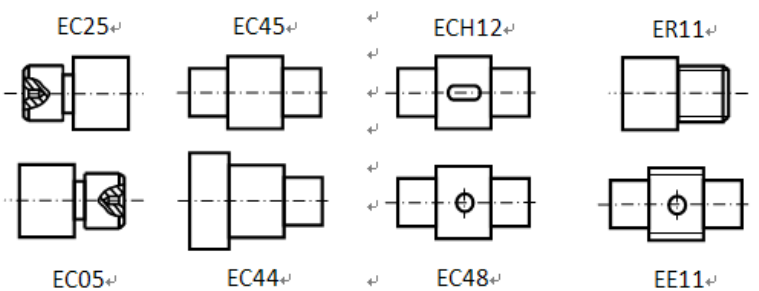

Figure 5. Representation of the primitive form features (PFF) used in this research. 


\section{DISCUSSION}

The value of global similarity metric for the case CASE01 equal to 0.92, near unity, indicates higher similarity to the new piece that the study case CASE02. As could be proved in their construction drawings shown in Figures 2 and 4, both parts are of equal value ICP-2D which leads to a strong similarity in the order of technological operations of both parts. But the differences between their PFF suggest changes to the type of operation and its technological steps.

In the elaboration of the process plan of the new part, it will be necessary to carry out the adding and eliminating of technological operations to the process plan of the case CASE01 to adapt it to the technological characteristics of the new part. This action corresponds to the adaptation phase in the cycle of CBR systems.

The lower similarity value achieved in the case CASE02 equal to 0.50 is justified by the no correspondence of their ICP-2D and the presence of different PFF in both parts.

\section{CONCLUSIONS}

This research has arrived at the following conclusions:

A function of global similarity metric to compare axisymmetric solid parts was obtained, that contains as traits local similarity, the geometric shape and characteristics of the stock in CAPP systems by Case Based Reasoning (CBR).

The traits local similarity for stock incorporates, in addition to the attributes of the part normally used, the type of material, the type of stock and the relationship of the length-diameter dimensions of the part (L/D).

The case CASE01 showed the highest value of global similarity with the new part equal to 0.92 , based primarily on having the same value of ICP-2D, the difference between the two parts is marked by the no correspondence between two of its PFF.

The value of ICP-2D is associated with the similarity of Technological Route which will follow the parts being compared, while the value of IFG index considers the differences in the content of technological operations.

\section{REFERENCES}

[1] Rich E. Inteligencia Artificial - Barcelona: Gustavo Gili, S.A, 1988.

[2] Castillo E and Gutiérrez J M Expert Systems and Probabilistic Network Models, Springer, Versión Española editada por la Academia Española de Ingeniería. - New York, 1997.

[3] Hilera J and Martínez V. Redes Neuronales Artificiales: Fundamentos, modelos y aplicaciones - Madrid, España: AddisonWesley, 1995.

[4] Pawan Sharma, Mahendra Sharma. "Artificial Intelligence in Advance Manufacturing Technology-A Review Paper on Current Application”. International Journal of Engineering, Management \& Sciences (IJEMS) ISSN-2348 -3733, Volume-1, Issue-1, January 2014

[5] S. M. Seyedhosseini, A. Makui, M. Ghadami “Applying Case-Based Reasoning in Supporting Strategy Decisions” World Academy of Science, Engineering and Technology, Vol: 5, 2011

[6] Kolodner J.L: Memory for Experience. The Psychology of Learning and Motivation, Academic Press / ed. Editor Bower G. - Orlando, Florida, USA, 1985. - Vol. 9.
[7] Jurisica I: Representation and Management Issues for Case Based Reasoning Systems, Artificial Intelligent Magazine. - USA: 1993. - 7: Vol. 8. - pp. 28-34.

[8] Pearl P: Issues in Case-Base Design Systems, - England: AI-EDAM, 1993. 2: Vol. 7. - pp. 79-85.

[9] Schank R C, Dynamic Memory: A theory of learning in computers and peoples - New York, USA: Cambridge University Press, 1995.

[10] Watson, I. "Case-Based Reasoning is a methodology not a technology”. Knowledge-Based Systems. (1999), 303-308.

[11] Aamodt A and Plaza E, Case-Based Reasoning: Foundational issues, methodological variations, and system approaches. : AI Communications, 1994. Vol. 7. - pp. 39-59.

[12] Hao Yang, Wen F. Lu and Alan C. Lin: "PROCASE: a case-based process planning system for machining of rotational parts”. Journal of Intelligent Manufacturing. (1994), 411-430.

[13] Muñoz-Avila H. "Integrating Twofold Case Retrieval and Complete Decision Replay in CAPLAN/CBC”. Tesis Doctoral. Universität Kaiserslautern, mayo, (1998), 198.

[14] Zuntong W., Zhanqiang L., and Xing A., “A Case representation and similarity in high-speed machining”. Elsevier. DOI:10.1016/S0890-6955(03)00152-4. (2003), 1347-1353.

[15] Hsin-chi C., Wen F. L., and Xiaoqing F. L.: "Intelligent Case Retrieval and Modification for Machining Process Planning of Axisymmetric Parts”. Proceedings of the Artificial Intelligence and Manufacturing (1998). 